\title{
Combined Microbial and Tissue-Mediated Induction as A Potentiality for Staged Carcinogenesis and Spread of Colo-Rectal Cancer
}

\author{
Lawrence M Agius* \\ Department of Pathology, University of Malta Medical School, Malta Europe
}

Submission: February 14, 2019; Published: March 19, 2019

"Correspondence Author: Lawrence M Agius, Department of Pathology, Mater Dei Hospital, Tal-Qroqq, University of Malta Medical School, Msida, Malta, Europe

\begin{abstract}
The phenomenal re-evolution of events as primarily staged induction is cooperative dimension within integral emergence of the metastatic cancer spread in terms of successive cycles of induced adaptability and transformation. Incremental dimensions include staged metastatic spread as staged carcinogenesis as well defined in Colo-rectal cancer. The evolution of staged participation lies within confines of a genetic program that participates initially with infiltration of the colon wall and further concludes as organ deposition. Progression of cancer growth and spread are therefore determinants of a genetic dysfunctional in terms largely of an inducing phenomenon of infiltrative spread. Both microbial and tissue mediation of colorectal carcinogenesis are organised in a contextual stratification that promotes and further enhances the emergence of genetic lesions and the repair damage response that yield dimensions of excessive growth and malignant transformation as carcinogenesis in the colon or rectum.
\end{abstract}

\section{Introduction}

Epidemiological and experimental evidence have indicated that colorectal cancer is closely related to lifestyle, especially diet [1]. Due to the common occurrence of colo-rectal cancer as a cancer-related cause of death, the dynamics and progression of this type of malignancy indicate a systematic reproduction/ turnover of tumor within the specific evolutionary pathways of a lesion induced by both acquired and hereditary genetic factors. Inhibitor of growth family 5 appears implicated in cell regeneration and colorectal carcinogenesis, and in DNA repair, apoptosis proliferation inhibition and chromatin remodeling and infiltration [2]. In view of such dimensions, it is further realized that the initiation and further development of malignant evolution is sufficient cause for the emergence of systemic pathway emergence within the continuous growth and spread of cells initially localized to the colon or rectum. The crypt cells in the colon are abnormally proliferative in a manner that defines the dysplastic changes that concurrently evolve with the establishment of marked proliferative potential. It is indeed within the phenomenon of establishment of such proliferating dysplastic crypt cells of the colonic mucosa that the further emergence of carcinomatous biologic traits induces collective and integral expression of dynamics of growth within the given strict dimension of spread of the emerging malignant cells.
Sonic Hedgehog, Wht wingless-type mouse mammary tutor ivrus/Beta-catenin, transforming growth factor-beta/SMAD, epidermal growth factor receptor and Notch are implicated in such colorectal tumor progression [3].

\section{Transformation Biology}

The substantial attribute as biology of the transformation process allows a strict implementation of systems of infiltrative potential within additional dimension of staged spread into the large bowel wall. It is beyond such concepts, however, that growth and spread of the malignant cells are staged potential acquisition in terms that justify the prognostic implications of Dukes' classification of such staging. Deficiency in STAT1 signaling promotes gut inflammation and colorectal carcinogenesis [4]. It is to be realized that fundamental resumption of growth as expressive attribute of tumor spread and metastatic deposits define the integral participation of multiple tissue pathways within such evolving characteristics as hepatic and pulmonary metastases. Colorectal cancer-derived small extracellular vesicles establish an inflammatory premetastatic niche for liver metastases [5].

Performance integers include the progression of tumor spread as dictated by lymph node and blood-system 


\section{Cancer Therapy \& Oncology International Journal}

determination of such spread. It is further to such considerations that hereditary traits of evolution determine and re-define the status of environmental and acquired dimensions of biologic definition of tumor recurrence or of tumor advancement that translates the cellular proliferation as determined system pathobiology. Epigenetic silencing of tumor suppressor genes is a central hallmark of colorectal carcinogenesis [6]. The reversible and dynamic nature of epigenetic modifications provides a promising strategy in colorectal cancer chemoprevention and treatment [7].

It is clearly within the classification of operative staging that there emerges the transforming attribute for further acquisition of malignant traits in tumor spread. Direct induction of DNA cross-links by a bacterium facilitates the delineated role of E.coli in colon cancer; DNA cross-links are severely mutagenic and profoundly cytotoxic lesions [8].

\section{Familial and Acquired Potentiality}

Familial adenomatous polyposis coli and hereditary nonpolyposis colon cancer are system pathways that indicate a central role for APC as gatekeeper for colo-rectal carcinogenesis in a defining system of acquisition that is strictly staged progression of malignant spread. Defined attributes of loss of genetic mutability or of acquired carcinogenicity is further dimension within the systemic lymphatic and blood-borne predetermination of spread of the malignant cells.

The significance of staged phenomena of spread of colorectal carcinogenesis indicates a profile occurrence that includes the defined attributes of colon/rectal cancer spread in terms of dimensional acquisition of abnormal potential towards determined emergence of multi-system evolution. Adiponectin is an adipokine with beneficial metabolic and anti-inflammatory properties; it exerts inhibitory effects on viability and migration of human colorectal cell lines [9].

The further cooperative operability of such dimensional characterization includes advanced participation of lymphatic and blood vessels that introduce circulatory dynamics within the actual acquisition of progressive active transformation in spread and in deposition of malignant cells within the body systems. The DNA damage response pathway maintains genetic integrity and effects cell cycle arrest, apoptosis, and senescence together with the induction of natural killer group 2 member D ligands on the surface of stressed cells [10]

\section{Staged Apposition}

Staged re-appraisal allows for integral participation of multi-system cooperativity within the reduction phenomena of metastatic spread in terms of progressive carcinogenesis. Commensal-polarised macrophages induce gene mutation, chromosomal instability, and endogenous transformation through microbiome-induced bystander effects, and thus the initiation of colorectal carcinogenesis [11). Included parameters allow for a permissive re-dimensionalization in the distributional assortment of dynamics of cancer spread. Indeed, the specific metastasizing dimensions of primary colo-rectal cancer is developmental and inductive within the varied pathways for emergence of malignant change as differential progression of the primary carcinogenesis pathways themselves that in turn yield the phenomenon of differentiation in the hereditary/acquired apposition of system spread.

\section{Cycles of Cellular/Tissue Induction}

Primary and secondary subsequent cycles of re-induction are hence evolutionary pathways that determine the progression not only of spread and metastatic deposition, but also of the integral carcinogenesis pathways giving rise to the initially emerging malignant lesion in the colon or rectum. In such definition, the conceptual realization of events as strictly evolutionary is re-defined as integral participation of stem cell biology within the colonic glandular crypts. Cancer stem cells are the focus of study in a range of cancers including colorectal cancer with the identification of genes in the induction and maintenance of pluripotency of stem cells and markers for cancer stem cells in colorectal cancer [12]. In such terms, the propagation of inducing pathways are central core determinants within the progression of the inducing phenomena themselves. Hence, in defined participation of malignant transformation of crypt cells, there is a system re-characterization of events in carcinogenesis that carries the potential appropriation of the malignant change as system cycles of adaptability and transformation. Changes in the intestinal microbiota composition in colorectal cancer patients compared to control subjects occur, with several bacterial species involved in pro-inflammation and pro-carcinogenesis [13]. Realization of genetic injury is specifically inductive and productive within the pathobiology of staged spread of the primary colonic or rectal carcinoma.

Performance dynamics hence are highly potential resources within the acquisition of cycles of induction as both hereditary and acquired dimensions of staged progression. The further emergence of such attributes further conforms to the characterization of events constituting staged carcinogenesis in further dimensional emergence of induction to spread and metastatic deposition. Toll-like receptors are implicated in transduction of molecular signals in immune induction and regulation, production of cytokines, and the recognition of specific molecular patterns on the surface of micro-organisms, and also in carcinogenesis in colorectal cancer as a staged phenomenon [14].

\section{Concluding Remarks}

The whole integral participation of hereditary and acquired pathways of staged induction of spread of the carcinomatous cells is indicative of a cyclic reformulation within the effective pre-determined potentiality for malignant cells. Clearly, the resultant growth and spread of malignant cells is a resultant 


\section{Cancer Therapy \& Oncology International Journal}

participation of repair genes that only approximately correct genetic lesions within the colon/rectal crypt cells. Microbeinduced oncogenicity is not a strict correlate of commensal virulence and can be evoked by even non-pathogenic E. coli by inducing tumorigenic stemness in host cells [15].

Indeed, further collaborative indices reduce staged participation in carcinogenesis as the essential event within progressive phases of involving systems of spread and metastatic deposition. It is likely that a re-appraisal of dynamics is inducing pre-determination that cooperatively reconstitutes genetic lesions in terms of approximating dimensions of induction and collaboration of carcinogenesis. Such carcinogenesis is indicative functionality within the stages of system participation in cancer spread.

\section{References}

1. Tamura S, Narita T, Fujii G, Miyamoto S, Hamoya T, et al. (2019) Inhibition of NF-kappaB transcriptional activity enhances fucoxanthinol-induced apoptosis in colorectal cancer cells Genes Environ 41: 1.

2. Yang XF, Shen DF, Zhao S, Ren TR, Gao Y, et al. (2019) Expression pattern and level of ING5 protein in normal and cancer tissues Oncol Lett 17(1): 63-68,

3. Tiwari A, Saraf S, Verma A, Panda PK, Jain SK (2018) Novel targeting approaches and signaling pathways of colorectal cancer: an insight World J Gastroenterol 24(39): 4428-4435.

4. Leon-Cabrera S, Vazquez-Sandoval A, Molina-Guzman E, DelgadoRamirez Y, Delgado-Buenrostro NL, et al. (2018) Deficiency in STAT1 signaling predisposes gut inflammation and prompts colorectal cancer development Cancers (Basel) 10(9)

5. Shao Y, Chen T, Zheng X, Yang S, Xu K, et al. (2018) Colorectal cancerderived small extracellular vesicles establish an inflammatory premetastatic niche in liver metastasis. Carcinogenesis 3(11): 13681379.
6. Gerecke C, Schumacher R, Edich A, Wetzel A, Yealland G, et al (2018) Vitamin C promotes decitabine or azacytidine induced DNA hydroxymethylation and subsequent reactivation of the epigenetically silenced tumour suppressor CDKN1A in colon cancer cells. Oncotarget 9(67): 32822-32840.

7. Zuo Q, Wu R, Xiao X, Yang C, Yang Y, et al. (2018) The dietary flavone luteolin epigenetically activates the Nrf2 pathway and blocks cell transformation in human colorectal cancer HCT116 cells J Cell Biochem 119(11): 9573-9582.

8. Bossuet-Greif N, Vignard J, Taieb F, Mirey G, Dubois D (2018) The Colibactin genotoxin generates DNA interstrand cross-links in infected cells. MBio 9(2)

9. Nigro E, Schettino P, Polito R, Scudiero O, Monaco ML (2018) Adiponectin and colon cancer: evidence for inhibitory effects on viability and migration of human colorectal cell lines. Mol Cell Biochem 448(1-2): 125-135

10. Espinoza JL, Minami M (2018) Sensing bacterial-induced DNA damaging effects via natural killer group 2 member D immune receptor: from dysbiosis to autoimmunity and carcinogenesis Front Immune 9: 52 .

11. Wang X, Yang Y, Huycke MM (2017) Commensal-infected macrophages induce dedifferentiation and reprogramming of epithelial cells during colorectal carcinogenesis. Oncotarget 8(60): 102176-102190.

12. Munro MJ, Wickremesekera SK, Peng L, Tan ST, Itinteang T (2018) Cancer stem cells in colorectal cancer: a review. J Clin Pathol 71(2): 110-116.

13. Lucas C, Barnich N, Nguyen HTT (2017) Microbiota, inflammation and colorectal cancer. Int J Mol Sci18(6).

14. Bednarczyk M, Muc-Wierzgon M, Walkiewicz K, Kokot T, Fatyga E, et al. (2017) Profile of gene expression of TLR-signaling pathways in colorectal cancer tissues. Int J Immunopathol Pharmacy 30(3): 322326.

15. Sahu U, Choudhury A, Parvez S, Biswas S, Kar S (2017) Induction of intestinal stemness and tumorigenicity by aberrant internalisation of commensal non-pathogenic E. coli. Cell Death Dis 8(3).

\section{Your next submission with Juniper Publishers will reach you the below assets}

- Quality Editorial service

- Swift Peer Review

- Reprints availability

- E-prints Service

- Manuscript Podcast for convenient understanding

- Global attainment for your research

- Manuscript accessibility in different formats ( Pdf, E-pub, Full Text, Audio)

- Unceasing customer service

Track the below URL for one-step submission https://juniperpublishers.com/online-submission.php 